\title{
A Wolbachia triple-strain infection generates self-incompatibility in Aedes albopictus and transmission instability in Aedes aegypti
}

Thomas H. Ant ${ }^{1,2,3}$ and Steven P. Sinkins ${ }^{1,2^{*}}$

\begin{abstract}
Background: Artificially-introduced transinfections of the intracellular bacterium Wolbachia pipientis have the potential to reduce the vectorial capacity of mosquito populations for viruses such as dengue and chikungunya. Aedes albopictus has two native strains of Wolbachia, but their replacement with the non-native wMel strain blocks transmission of both viruses. The pattern of cytoplasmic incompatiiblity generated by wMel with wild-types is bidirectional. Novel-plus-native-strain co-infection is predicted to lead to a more efficient population spread capacity; from a bi-directional to a uni-directional cytoplasmic incompatibility (Cl) model.

Results: A novel-plus-native-strain triple-infection in Ae. albopictus (wAlbAwAlbBwMel) was generated. Although triple-infected females were fully reproductively viable with uninfected males, they displayed self-incompatibility. qPCR of specific strains in dissected tissues suggested that this may be due to the displacement of one of the native strains (wAlbA) from the ovaries of triple-infected females. When the triple strain infection was transferred into Aedes aegypti it displayed an unexpectedly low level of transmission fidelity of the three strains in this species.

Conclusions: These results suggest that combining Wolbachia strains can lead to co-infection interactions that can affect outcomes of $\mathrm{Cl}$ and maternal transmission.
\end{abstract}

Keywords: Wolbachia, Aedes albopictus, Aedes aegypti, Superinfection, Cytoplasmic-incompatibility, Populationreplacement

\section{Background}

Wolbachia pipientis is a maternally transmitted bacterial endosymbiont that is naturally carried by a broad range of terrestrial arthropods. The generation of novel Wolbachia transinfections in mosquitoes can result in reduced host permissivity for a range of pathogens [1-10]; for example, Aedes albopictus females transinfected with the Wolbachia strain $w \mathrm{Mel}$ do not transmit dengue [7] or chikungunya [8] viruses in laboratory challenges. Wolbachia can invade host populations by increasing the relative fitness of infected females through a pattern of crossing sterility known as cytoplasmic incompatibility

\footnotetext{
*Correspondence: steven.sinkins@glasgow.ac.uk

${ }^{1}$ Centre for Virus Research, University of Glasgow, Glasgow, UK

${ }^{2}$ Biomedical and Life Sciences, Lancaster University, Lancaster, UK

Full list of author information is available at the end of the article
}

(CI) [11]. CI occurs when a male infected with a Wolbachia strain mates with either an uninfected female or a female carrying a different non-compatible Wolbachia strain, and results in fertilization but inviability of the developing embryo. CI can be categorised according to whether populations show uni- or bi-directional sterility. In the simplest form of unidirectional CI, Wolbachiacarrying females are fully compatible with both Wolbachia-infected and Wolbachia-naive males, but naïve females are compatible with naive males only. Wolbachia-carrying females therefore do not suffer the fitness costs resulting from incompatible matings.

The fitness advantage of CI for Wolbachia-carriers increases with population infection frequency (with uninfected females experiencing higher proportions of incompatibility), although fitness benefits may still be

(c) The Author(s). 2018 Open Access This article is distributed under the terms of the Creative Commons Attribution 4.0 International License (http://creativecommons.org/licenses/by/4.0/), which permits unrestricted use, distribution, and 
appreciable at low frequencies. In the case of bidirectional CI, two interbreeding populations carry reciprocally incompatible Wolbachia infections; males and females of each infection type only producing viable progeny with carriers of the same infection. Females infected with the lower frequency strain will experience a higher proportion of incompatibility and will tend towards lower relative fitness. Spread through bidirectional $\mathrm{CI}$ is therefore typically associated with higher invasion threshold frequencies compared to unidirectional CI.

Crosses involving hosts carrying Wolbachia superinfections have shown that the effects of separate CI modifications can be additive [12-14]. Unidirectional CI can be produced when a superinfected line is crossed with a population carrying constituent strain(s) of the superinfection. Achieving population replacement with $\mathrm{Wol}$ bachia necessitates the release of infected female carriers; for public acceptance, logistical and economic reasons, it is desirable to release as few Wolbachia-carrying mosquitoes as possible to achieve self-sustaining spread. As minimum threshold frequencies for successful population invasion are typically lower under a unidirectional CI model, the generation of superinfections in naturally infected species consisting of a novel Wolbachia transinfection in addition to any native strains should allow for population replacement with fewer releases. Moreover, superinfected strains may allow for higher intracellular densities to be achieved than with a single constituent strain, resulting in improved pathogen inhibition. Joubert and colleagues [15] reported the generation of a superinfection in Aedes aegypti in which the $w \mathrm{Mel}$ and $w \mathrm{AlbB}$ Wolbachia strains were combined, and found more robust dengue virus inhibition in the superinfected strain compared to infections with the single constituent strains. The $w$ MelwAlbB line also showed a strong uni-directional CI crossing pattern with both $w \mathrm{Mel}$ and $w$ AlbB-only carriers, suggesting that the $w$ Mel $w$ AlbB superinfection would be able to drive through single-infected populations, and could potentially rescue a control programme in the event that the dengue-blocking effectiveness of a particular strain diminished over time. Superinfections also allow the potential mixing of desirable strain phenotypes; a $w \mathrm{Au}-$ $w$ AlbB double-infection in Ae aegypti combined the robust pathogen blocking capacity of Wolbachia strain $w \mathrm{Au}$ with the strong $\mathrm{CI}$ induction of $w$ AlbB [10].

We attempted to create a native plus novel strain triple superinfection in Aedes albopictus, a highly invasive dengue, chikungunya and Zika virus vector with a largely suburban/semi-rural distribution. Aedes albopictus is naturally superinfected at high prevalence with two Wolbachia strains termed wAlbA and wAlbB, with $w$ AlbA existing at approximately $10 \%$ the intracellular density of wAlbB [16]. Aedes albopictus was previously cured of its native Wolbachia infections [17] and transinfected with $w \mathrm{Mel}$ from Drosophila melanogaster; this line showed strong reductions in the transmission of dengue [7] and chikungunya [8] viruses. The $w$ Mel infection also showed bidirectional CI with wild-type ( $w$ AlbAwAlbB) infected mosquitoes [7]. Here we report the generation of a $w$ MelwAlbAwAlbB triple superinfection in Ae. albopictus and assess its stability, crossing types and intracellular densities. We also describe the transfer of the triple superinfection to Ae. aegypti by embryonic microinjection, and assess its stability in this species.

\section{Results}

\section{Superinfection generation and maternal transmission in Ae. albopictus}

A triple strain superinfection comprising the native strains $w \mathrm{AlbA}, w \mathrm{AlbB}$ and a novel strain $w$ Mel was generated in Ae. albopictus (hereon, wAlbAwAlbBwMel) through the transfer of cytoplasm from a $w \mathrm{Mel}$ infected Ae. albopictus line into wild-type (wAlbAwAlbB-carrying) embryos. Aedes albopictus wAlbAwAlbBwMel females were outcrossed to Wolbachia-negative males for three generations. At $\mathrm{G}_{4}$ maternal inheritance rates were assessed by crossing females of the triple-infected line with Wolbachia-negative males, and the resulting progeny were assessed for Wolbachia infection status by strain-specific PCR. The $w A l b A w A l b B w$ Mel line showed high rates of maternal transmission of all three Wolbachia strains to progeny: of 100 individuals tested, 97 contained all three strains while 3 carried $w$ Mel only.

\section{$\mathrm{Cl}$ crossing types in Ae. albopictus}

In order to determine patterns of $\mathrm{CI}$, crosses were set-up between triple-infected, wild-type and Wolbachia-negative mosquitoes. Crosses involving males of the wAlbA$w \mathrm{AlbB} w$ Mel line resulted in fully penetrant $\mathrm{CI}$ with wild-type and Wolbachia-negative Ae. albopictus females, with no eggs hatching from either cross (Table 1). When females of the $w$ AlbAwAlbBwMel infected line were crossed to $w \mathrm{AlbA} w \mathrm{AlbB} w \mathrm{Mel}$ males, the embryos also showed very low hatch rates, in other words the line displayed self-incompatibility. Females of the wAlbA$w \mathrm{AlbB} w \mathrm{Mel}$ line were able to produce viable eggs when mated to Wolbachia-negative males and could successfully rescue (effectively overcome) the CI modification of $w$ Mel-only males, but showed reduced hatch rates when crosses to wild-type males. This suggests that either (or both) of the wAlbA or $w$ AlbB sperm modifications were causing $\mathrm{CI}$ in the progeny of triple-infected females.

\section{Wolbachia densities in Ae. albopictus wAlbAwAlbBwMel}

The overall Wolbachia densities, and the densities of the individual Wolbachia strains were assessed in whole adult females and in dissected ovary, midgut and salivary 
Table 1 Percentage hatch rates ( \pm SD) of eggs resulting from crosses between Ae. albopictus lines

\begin{tabular}{lllll}
\hline & & & \\
\cline { 2 - 5 } & wAlbAwAlbB & wAlbAwAlbBwMel & wMel & \\
\hline wAlbAwAlbB & $92.1 \pm 4.2$ & 0 & 0 & - ve \\
wAlbAwAlbBwMel & $7.0 \pm 6.7$ & $5.8 \pm 4.6$ & $67.5 \pm 13.8$ & $65.2 \pm 3.2$ \\
wMel & 0 & 0 & $73.7 \pm 5.6$ & $77.9 \pm 12.1$ \\
-ve & 0 & 0 & 0 & $88.5 \pm 4.0$ \\
\hline
\end{tabular}

Each percentage shows the mean hatch rates from eggs resulting from three separate cages, each containing ten females and twenty males. For each cage $>420$ eggs were assessed

gland tissues by qPCR. The overall adult female Wolbachia densities in the triple-infected $w \mathrm{AlbA} w \mathrm{AlbB} w \mathrm{Mel}$ line were found to be significantly higher than in the wild-type (one-way ANOVA, $F_{(1,19)}=31.8, P=0.00024$ ) (Fig. 1a), consistent with previous findings of the $w$ Melonly infection reaching high densities in Ae. albopictus [7]. In whole females, the density of the $w$ AlbB infection appeared to be unaffected by the presence of $w \mathrm{Mel}$, while $w$ AlbA showed a non-significant reduction (oneway ANOVA, $\left.F_{(1,19)}=3.8, P=0.07\right)$ (Fig. 1b).

In ovary tissues, no reduction was found in the density of $w \mathrm{AlbB}$ in the superinfected line compared to wild-type, while the density of $w$ AlbA was significantly reduced (from $19.1 \pm 10.8$ to $5.8 \pm 3.2$ Wolbachia/host genome copies, mean \pm SD; one-way ANOVA, $F_{(1,19)}=13.51, P=$ 0.0017 ) (Fig. 2a). The reduced $w$ AlbA ovary density is a potential cause of the observed self-incompatibility - with density remaining high enough for maternal transmission to occur, but insufficient in embryos to allow full rescue of the $w$ AlbA CI sperm modification.

As the fitness of host and Wolbachia depends on maximising mosquito reproductive output, it is expected that co-evolutionary pressures will favour native Wolbachia localisation to the germline, and restrict densities in tissues not directly involved in vertical transmission (where high densities can lead to reductions in host fitness). Consistent with this, the native $w \mathrm{AlbA}$ and $w \mathrm{AlbB}$ strains were found to be localised largely in the ovaries (Fig. 2a), while the novel wMel infection showed a far more diffuse tissue distribution, reaching relatively high densities in the somatic tissues of the midgut (Fig. 2b) and salivary glands (Fig. 2c).

\section{Triple-infection transfer and maternal transmission in Ae. aegypti}

Cytoplasm from embryos of the triple-infected Ae. albopictus line was transferred to embryos of wild-type Ae. aegypti by microinjection. $G_{0}$ females were out-crossed to wildtype males, blood-fed and individualised for oviposition. A single $G_{0}$ female produced $G_{1}$ progeny that were PCRpositive for all three Wolbachia strains (here-on Ae.aeg$w \mathrm{Mel} w \mathrm{AlbA} w \mathrm{AlbB})$. The female progeny of this line were out-crossed to wild-type males for three consecutive generations. Screening of progeny during this period revealed an unexpectedly high instability in maternal transmission of the triple infection, with progeny containing various combinations of strains recovered at high rates. At $\mathrm{G}_{4}$, females backcrossed to wild-type males were blood-fed and were individualised for oviposition. Individualised females were assessed for infection by PCR, and the eggs of confirmed triple-infected females were selected (20 in total). Eggs were hatched and a sample of 10 L4 larval progeny from each female were assessed for Wolbachia infection status by strain-specific PCR (200 larvae in total). Individuals
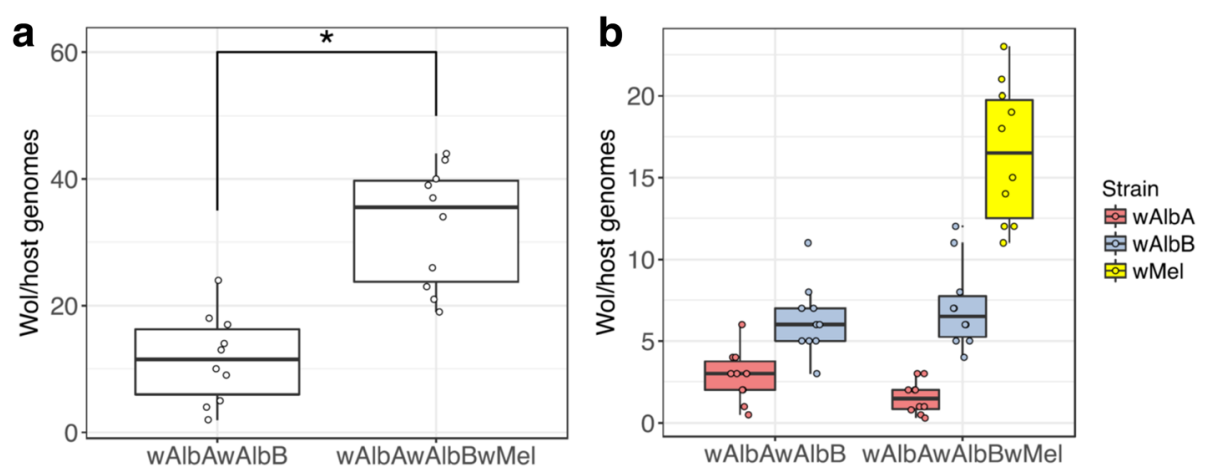

Fig. 1 Densities of Wolbachia in adult Ae. albopictus females, measured by qPCR. Total Wolbachia densities (a) and strain-specific densities (b) in whole adult females. The centre of a box plot shows median Wolbachia density, edges show upper and lower quartiles, and whiskers indicate upper and lower extremes. Dots show values from individual biological replicates 

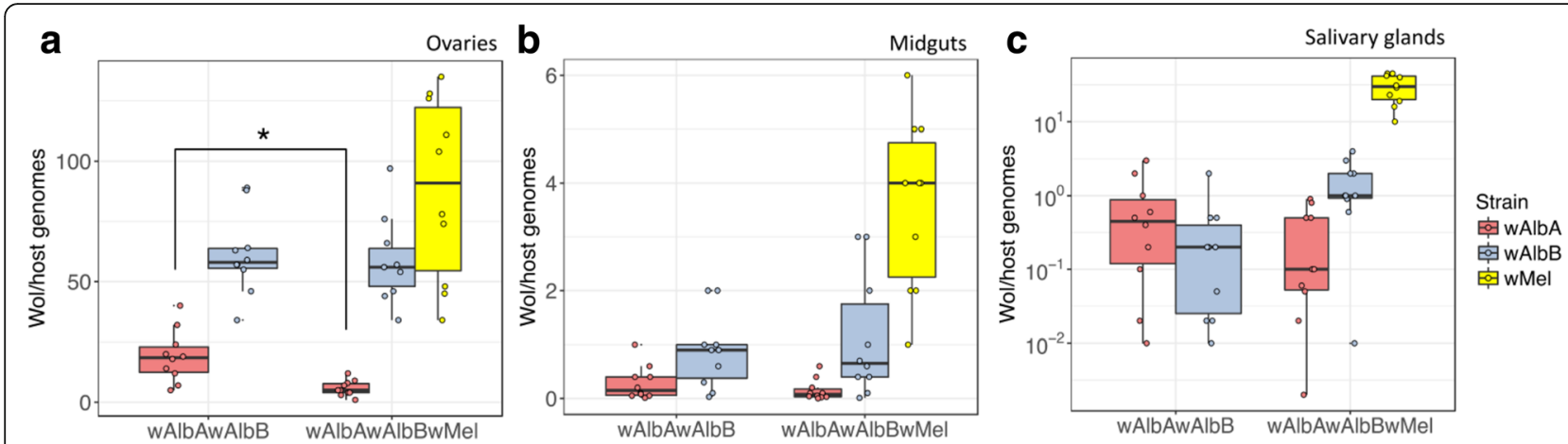

Fig. 2 Strain-specific Wolbachia densities in dissected tissues. Panels show densities in ovaries (a), midguts (b) and salivary glands (c). Each box represents 10 biological replicates, with pools of 5 females or the tissues from 5 females per replicate. The centre of a box plot shows median Wolbachia density, edges show upper and lower quartiles, and whiskers indicate upper and lower extremes. Dots show values from individual biological replicates

carrying wAlbB-only, wMel-only, and superinfections of $w$ Mel $w$ AlbB, $w$ Mel $w$ AlbA, $w$ AlbA $w$ AlbB and $w$ Mel $w A l b A-$ $w$ AlbB were recovered at varying rates (Table 2).

The Ae. aegypti wAlbAwAlbB line was out-crossed to wild-type males and displayed complete $(100 \%)$ maternal transmission of both strains over a large number of generations (screening > G10), suggesting that the presence of $w$ Mel was the cause of the observed transmission infidelity in the triple-strain superinfected line.

\section{Aedes aegypti wMelwAlbAwAlbB crossing types}

A series of individual male and female single crosses were set up involving the triple-infected and wild-type mosquitoes. After eggs from single crosses were obtained, the infection status was assessed by PCR and only the eggs from crosses involving PCR-confirmed triple infected individuals were hatched and used in assessing crossing types.

When triple-infected males were crossed to wild-type females, full CI was observed, with no eggs hatching. Low hatch rates were observed when triple-infected males and triple-infected females were crossed $(21.3 \pm 12.4 \%)$, in comparison to $76.5 \pm 9.2 \%$ when wild-type males were

Table 2 Maternal transmission fidelity of Wolbachia strains in Ae.aeg-wAlbAwAlbBwMel

\begin{tabular}{ll}
\hline Wolbachia strain(s) & $\%$ (Frequency) \\
\hline wAlbB & $11(22 / 200)$ \\
wMel & $21(42 / 200)$ \\
wAlbBwMel & $2(4 / 200)$ \\
wAlbAwMel & $6.5(13 / 200)$ \\
wAlbAwAlbB & $14(28 / 200)$ \\
wAlbAwAlbBwMel & $45.5(91 / 200)$
\end{tabular}

Eggs from crosses between triple-infected females and wild-type males were hatched and a proportion of randomly selected L4 larvae were screened for infection status by Wolbachia strain-specific PCR. Numbers show percentage frequency and parenthesis provide actual numbers crossed to triple-infected females, and $87.4 \pm 4.1 \%$ for wild-type male and female control crosses (Table 3). Given incomplete maternal transmission, the low hatch rates in the triple-infected male and female within-line cross may result from either self-incompatibility, or incompatibility between triple infected males and progeny of triple infected females that do not inherit all three Wolbachia strains - or some combination of both. Less than half of eggs receive the full complement of Wolbachia strains (Table 2), and are therefore expected to experience incompatibility with sperm resulting from a triple-infected male.

The crossing types of the Ae. aegypti $w \mathrm{AlbA} w \mathrm{AlbB}$ infection with the constituent $w$ AlbA, $w$ AlbB and wild type strains was assessed and showed full uni-directional CI with each (Table 4), and was self-compatible, as would be predicted. This lends support to the hypothesis that the presence of $w \mathrm{Mel}$ that was causing the unexpected crossing patterns in the triple-infected line.

\section{Discussion}

The wMel-only infection in Ae. albopictus was previously reported to produce bidirectional CI with wildtype mosquitoes [7], but provided strong pathogen inhibition against the dengue [7] and chikungunya (8) viruses with minimal effects on host fitness [8]. We created a triple-infected line combining $w$ Mel with the native strains $w \mathrm{AlbA}$ and $w \mathrm{AlbB}$, with the aim of

Table 3 Percentage hatch rates ( \pm SD) of eggs resulting from crosses between Ae. aegypti lines

\begin{tabular}{llll}
\hline & & \multicolumn{2}{l}{} \\
\cline { 3 - 4 } & & wMelwAlbAwAlbB & wt \\
\hline+ & wMelwAlbAwAlbB & $21.3 \pm 12.4$ & $76.5 \pm 9.2$ \\
& wt & 0 & $87.4 \pm 4.1$ \\
\hline
\end{tabular}

Each percentage shows the mean hatch rates from eggs resulting from ten separate single crosses, each containing a single female and a single male. For each cross $>50$ eggs were assessed 
Table 4 Crossing types of Ae. aegypti infected with either wAlbA-only, wAlbB-only, a superinfection of wAlbA and wAlbB, or uninfected (wt)

\begin{tabular}{llllll}
\hline & & \multicolumn{3}{c}{} \\
\cline { 3 - 6 } & & wt & wAlbA & wAlbB & wAlbAwAlbB \\
\hline \hline & wt & 83.2 & 0 & 0 & 0 \\
& wAlbA & 81.7 & 77.4 & 0 & 0 \\
& wAlbB & 80.4 & 0 & 84.3 & 0 \\
& wAlbAwAlbB & 71.9 & 75.5 & 72.3 & 78.4 \\
\hline
\end{tabular}

Numbers show mean hatch rates, with $>250$ eggs counted for each cross

creating a wMel-carrying line that produces unidirectional CI with wild populations, and is thus expected to have improved population invasion ability. While males of the triple infected $w \mathrm{AlbA} w \mathrm{AlbB} w \mathrm{Mel}$ line were able to successfully induce CI when crossed with wild-type females, the $w$ AlbAwAlbB $w$ Mel line showed selfincompatibility. The $w$ AlbA $w$ AlbB $w$ Mel females could successfully rescue $\mathrm{CI}$ from $w$ Mel-only males, but were incompatible with wild-type males, suggesting an inability to rescue CI from $w$ AlbA and/or $w$ AlbB. The observation of significantly reduced densities of $w$ AlbA in $w \mathrm{AlbA} w \mathrm{AlbB} w \mathrm{Mel}$ ovaries compared to wild-type suggests that wAlbA in embryos reaches too low a density to be able to rescue the $\mathrm{CI}$ modification produced by the $w$ AlbA strain in the males.

The self-incompatibility of the wMelwAlbAwAlbB superinfection in Ae. albopictus means that it is unlikely to be a useful alternative for dengue control, and the $w$ Mel-only Ae. albopictus line remains the best option for dengue transmission blocking in this species. Selfincompatibility will represent a potential natural barrier to new strains of Wolbachia successfully transferring into a host species that already contains one or more Wolbachia strains, if there are competitive interactions that reduce the density of one of the strains already present. The $w$ Mel infection in the triple-infected $A e$. albopictus line showed a wider tissue distribution compared to the native strains, which were strongly localised to the ovaries. This finding lends support to the hypothesis that co-evolutionary selective pressures favour the localisation of native Wolbachia infections to the germline over time.

Several native plus novel strain triple infections have previously been reported in Ae albopictus. A tripleinfection consisting of $w \mathrm{AlbA}, w \mathrm{AlbB}$ and $w \mathrm{Ri}$ showed full uni-directional $\mathrm{CI}$ with wild-type mosquitoes and stable maternal transmission [14]. The difference in CI crossing patterns between the triple infections carrying either $w \mathrm{Ri}$ or $w \mathrm{Mel}$ may be a result of the high ovarian densities reached by $w \mathrm{Mel}$, leading to higher levels of native strain exclusion. This suggests that an important consideration when generating further superinfected lines will be the selection of novel strains with minimal impacts on native strain density. In addition, Ae. albopictus triple infections have been generated carrying $w$ AlbA, $w$ AlbB and $w$ Pip [18] and $w$ AlbA, $w$ AlbB and $w$ MelPop [19]. In the case of the triple infection containing $w$ MelPop, incomplete maternal transmission was observed when mosquitoes were fed a mouse-derived blood meal, with $w$ MelPop suffering particularly low transmission fidelity. Moreover, eggs from wMelPopcontaining triple-infected females displayed very low hatch rates in crosses to both triple-infected and wildtype ( $w$ AlbAwAlbB-carrying) males. Hatch rates were considerably higher (although still relatively low) when human blood was used. The authors suggest that the low hatch rates with mouse blood may be due to egg mortality resulting from elevated levels of nutrient depletion in the triple-infected embryos. An alternative explanation, supported by the present study, is that the low hatch rates are due to $\mathrm{CI}$ resulting from a degree of native strain exclusion in the ovaries, with the lower densities exacerbated on mouse blood. In Drosophila simulans the introduction of a third Wolbachia strain to an already naturally double-infected colony resulted in a stable triple infection, with high rates of maternal transmission and additive CI [20]. Naturally occurring triple infections have also been detected in the white fly, Bemisia tabaci [21], the adzuki bean beetle, Callosobruchus chinensis [22], and the parasitoid wasp, Trichogramma ostriniae [23].

We also transferred the triple infection into Ae. aegypti, where an unexpectedly high rate of Wolbachia strain transmission infidelity was observed. Given that the lines produced are all bidirectionally incompatible, the results presented here may indicate that an upper limit exists with certain strain combinations to the number of Wolbachia infections that can stably infect a line, particularly with novel hosts such as Ae. aegypti. This limit will likely be dependent on the densities of the infecting strains, and should be lower for multistrain infections involving high density strains due to competitive interactions.

\section{Conclusions}

This study presents results assessing the stability and population invasion potential for a $w \mathrm{AlbA} w \mathrm{AlbB} w \mathrm{Mel}$ triple Wolbachia infection in Ae. albopictus and Ae. aegypti. In Ae. albopictus the triple infection showed high rates of vertical transmission, but a self-incompatible $\mathrm{CI}$ phenotype, suggesting that the triple-infection would be unlikely to persist in wild populations. It is probable that the self-incompatible phenotype is a result of an observed displacement of the wAlbA strain in the ovaries of tripleinfected females, which could result in diminished CI rescue. In Ae. aegypti the triple infection showed remarkably 
low maternal transmission fidelity. These results highlight the importance of inter-strain interactions in determining Wolbachia superinfection stability and therefore utility in vector-control.

\section{Methods}

\section{Mosquito strains and rearing}

The Ae. albopictus wild-type line was the Ascoli strain colonized from San Benedetto del Tronto, Italy in 2006 by G. Favia and colleagues. The uninfected line the UjuT strain generated by tetracycline treatment [17]. All mosquito colonies were maintained at $27^{\circ} \mathrm{C}$ and $70 \%$ relative humidity with a 12-hour light/dark photocycle. Larvae were fed tropical fish pellets (Tetramin, Tetra, Melle, Germany) and adults were given access to a sucrose meal ad libitum. Blood meals were provided using a Hemotek artificial blood-feeding system (Hemotek, Blackburn, UK) using defribrinated sheep blood (TCS Biosciences, Botyl Claydon, UK). Eggs were collected by providing damp filter-paper (Grade 1 filter paper, Whatman plc, GE Healthcare, Coventry, UK) as an oviposition source and were desiccated for 5-10 days prior to hatching in water containing $1 \mathrm{~g} / \mathrm{l}$ bovine liver powder (MP Biomedicals, Santa Ana, California, USA).

\section{Generation of Wolbachia-infected lines}

The $w A l b A w A l b B w M e l$ line was generated by transferring cytoplasm from wMel-carrying Ae. albopictus embryos to wild-type (wAlbAwAlbB-carrying) $A e$. albopictus embryos. The Ae.aeg-wAlbAwAlbBwMel line was created by transferring cytoplasm from the triple infected (wAlbAwAlbBwMel) Ae. albopictus line to wildtype (Malaysian) Ae. aegypti. Microinjections were performed using methods described previously [7]. Female $G_{0}$ survivors were back-crossed to wild-type males, blood-fed and separated individually for oviposition. $G_{0}$ females were analysed for Wolbachia infection by strainspecific PCR (see Additional file 1: Table S1 for sequences) and eggs from $G_{0}$ females negative for the target transinfection were discarded. Eggs of positive females were hatched and $G_{1}$ 's were assessed for target transinfection $\mathrm{G}_{0}-\mathrm{G}_{1}$ germ-line transmission.

\section{$\mathrm{Cl}$ and maternal inheritance}

Rates of CI induction and rescue both with wild-type and Wolbachia-negative mosquitoes were assessed by crosses involving 20 males and 10 females in each of three separate cages. A blood meal was provided and females were individualised for oviposition. Eggs were collected on damp filter paper, which was subsequently desiccated for 5 days at $27{ }^{\circ} \mathrm{C}$ and $70 \%$ relative humidity. Eggs were counted and hatched in water containing $1 \mathrm{~g} /$ 1 bovine liver powder. Larvae were counted at the L2-L3 stage to provide hatch rates.
To assess rates of maternal inheritance, females from the Wolbachia transinfected lines were crossed to uninfected males in pools of 20 males and 10 females. A blood meal was provided and females were individualised for oviposition. Resulting eggs were hatched and DNA from a selection of the resulting pupae were analysed for specific Wolbachia strains by PCR.

\section{Wolbachia strain-specific PCR and density qPCR}

For PCR analysis, genomic DNA was extracted from mosquitoes using the Livak method. For primer sequences see Additional file 1: Table S1. For measurements of Wolbachia density by qPCR, genomic DNA was extracted from mosquitoes using phenol/chloroform. Unless stated otherwise, mosquitoes used in density experiments were adults 5-days post-pupal eclosion. gDNA was diluted to $100 \mathrm{ng} / \mu \mathrm{l}$ using a NanoDrop spectrophotometer (Thermo Scientific, Waltham, Massachusetts, USA). A BioRad CFX-96 real-time PCR detection system was used (Bio Rad, Hercules, California, USA) with 2x SYBR-Green mastermix (Biotool, Houston, Texas, USA). Total Wolbachia density was analysed by absolute quantification against a dilution curve of a vector containing single copies of the homothorax $(\mathrm{HTH})$ gene and Wolbachia surface protein (wsp).

To specifically quantify the $w \mathrm{AlbA}, w \mathrm{AlbB}$, and $w \mathrm{Mel}$ strains, the following primers were used: $w \mathrm{AlbA}$ QAdir1 and QArev2; wAlbB - 183F and QBrev2; wMel qMel-F and qMel-R. All were normalized against $\mathrm{HTH}$ copies. The following program was used to run the qPCRs: $95{ }^{\circ} \mathrm{C}$ for $5 \mathrm{~min}, 40 \times$ cycles of $95^{\circ} \mathrm{C}$ for $15 \mathrm{~s}$ and $60{ }^{\circ} \mathrm{C}$ for $30 \mathrm{~s}$, followed by a melt-curve analysis.

\section{Additional file}

Additional file 1: Table S1. Primer sequences. (DOCX $16 \mathrm{~kb}$ )

Acknowledgements

We thank C. Herd (University of Glasgow), V. Geoghegan, K. Stainton and C. Harris (Lancaster University) for discussions and technical assistance.

Funding

The study was supported by the Wellcome Trust (202888, 108508), the Medical Research Council (ZK/16-021), and the Biotechnology and Biological sciences Research Council (BB/K004506).

Availability of data and materials

All data presented in this manuscript are available from the corresponding author upon reasonable request.

\section{Authors' contributions}

TA and SS conceived and designed the study. TA generated the strains and conducted the experiments. TA and SS analysed the data and wrote the manuscript. SS supervised the study. Both authors read and approved the final version of the manuscript.

Ethics approval and consent to participate Not applicable. 


\section{Competing interests}

The authors declare that they have no competing interests.

\section{Author details}

${ }^{1}$ Centre for Virus Research, University of Glasgow, Glasgow, UK. ${ }^{2}$ Biomedical and Life Sciences, Lancaster University, Lancaster, UK. ${ }^{3}$ Present Address: Department of Disease Control, London School of Hygiene and Tropical Medicine, Keppel Street, London, UK.

Received: 20 February 2018 Accepted: 23 April 2018

Published online: 11 May 2018

\section{References}

1. Moreira LA, Iturbe-Ormaetxe I, Jeffery JA, Lu G, Pyke AT, Hedges LM, et al. A Wolbachia symbiont in Aedes aegypti limits infection with dengue, chikungunya, and Plasmodium. Cell. 2009;139:1268-78.

2. Walker T, Johnson PH, Moreira LA, Iturbe-Ormaetxe I, Frentiu FD, McMeniman CJ, et al. The wMel Wolbachia strain blocks dengue and invades caged Aedes aegypti populations. Nature. 2011;476:450-3.

3. Bian $G, X u Y, L u P, X i e Y, X i$ Z. The endosymbiotic bacterium Wolbachia induces resistance to dengue virus in Aedes aegypti. PLoS Pathog. 2010;6: e1000833.

4. Frentiu FD, Zakir T, Walker T, Popovici J, Pyke AT, van den Hurk A, et al. Limited dengue virus replication in field-collected Aedes aegypti mosquitoes infected with Wolbachia. PLoS Negl Trop Dis. 2014;8:e2688.

5. van den Hurk AF, Hall-Mendelin S, Pyke AT, Frentiu FD, McElroy K, Day A, et al. Impact of Wolbachia on infection with chikungunya and yellow fever viruses in the mosquito vector Aedes aegypti. PLoS Negl Trop Dis. 2012;6:e1892

6. Ye YH, Carrasco AM, Frentiu FD, Chenoweth SF, Beebe NW, van den Hurk AF, et al. Wolbachia reduces the transmission potential of dengue-infected Aedes aegypti. PLoS Negl Trop Dis. 2015;9:e0003894.

7. Blagrove MS, Arias-Goeta C, Failloux AB, Sinkins SP. Wolbachia strain wMe induces cytoplasmic incompatibility and blocks dengue transmission in Aedes albopictus. Proc Natl Acad Sci USA. 2012;109:255-60.

8. Blagrove MS, Arias-Goeta C, Di Genua C, Failloux AB, Sinkins SP. A Wolbachia wMel transinfection in Aedes albopictus is not detrimental to host fitness and inhibits chikungunya virus. PLoS Negl Trop Dis. 2013;7:e2152.

9. Kambris Z, Blagborough AM, Pinto SB, Blagrove MS, Godfray HC, Sinden RE, et al. Wolbachia stimulates immune gene expression and inhibits Plasmodium development in Anopheles gambiae. PLoS Pathog. 2010;6:e1001143.

10. Ant TH, Herd CS, Geoghegan V, Hoffmann AA, Sinkins SP. The Wolbachia strain wAu provides highly efficient virus transmission blocking in Aedes aegypti. PLoS Pathog. 2018;14:e1006815.

11. Turelli M, Hoffmann AA. Rapid spread of an inherited incompatibility factor in California Drosophila. Nature. 1991;353:440-2.

12. Dobson SL. Reversing Wolbachia-based population replacement. Trends Parasitol. 2003;19:128-33.

13. Dobson SL, Marsland EJ, Rattanadechakul W. Wolbachia-induced cytoplasmic incompatibility in single- and superinfected Aedes albopictus (Diptera: Culicidae). J Med Entomol. 2001;38:382-7.

14. Fu Y, Gavotte L, Mercer DR, Dobson SL. Artificial triple Wolbachia infection in Aedes albopictus yields a new pattern of unidirectional cytoplasmic incompatibility. Appl Environ Microbiol. 2010;76:5887-91.

15. Joubert DA, Walker T, Carrington LB, De Bruyne JT, Kien DH, NeT H, et al. Establishment of a Wolbachia superinfection in Aedes aegypti mosquitoes as a potential approach for future resistance management. PLoS Pathog. 2016; 12:e1005434.

16. Dutton TJ, Sinkins SP. Strain-specific quantification of Wolbachia density in Aedes albopictus and effects of larval rearing conditions. Insect Mol Biol. 2004;13:317-22.

17. Otsuka Y, Takoaka H. Elimination of Wolbachia pipientis from Aedes albopictus. Med Entomol Zool. 1997;48:257-60.

18. Zhang D, Zheng X, Xi Z, Bourtzis K, Gilles JR. Combining the sterile insect technique with the incompatible insect technique: I-impact of Wolbachia infection on the fitness of triple- and double-infected strains of Aedes albopictus. PLoS One. 2015;10:e0121126.

19. Suh E, Fu Y, Mercer DR, Dobson SL. Interaction of Wolbachia and bloodmeal type in artificially infected Aedes albopictus (Diptera: Culicidae). J Med Entomol. 2016. https://doi.org/10.1093/jme/tjw084.
20. Rousset F, Braig HR, O'Neill SL. A stable triple Wolbachia infection in Drosophila with nearly additive incompatibility effects. Heredity (Edinb). 1999;82:620-7.

21. Nirgianaki A, Banks GK, Frohlich DR, Veneti Z, Braig HR, Miller TA, et al. Wolbachia infections of the whitefly Bemisia tabaci. Curr Microbiol. 2003:47:93-101.

22. Kondo N, ljichi N, Shimada M, Fukatsu T. Prevailing triple infection with Wolbachia in Callosobruchus chinensis (Coleoptera: Bruchidae). Mol Ecol. 2002;11:167-80.

23. Song Y, Shen Z, Wang Z, Liu H. Triple infection of Wolbachia in Trichogramma ostriniae. Entomologia Sin. 2009;52:445-52.

\section{Ready to submit your research? Choose BMC and benefit from:}

- fast, convenient online submission

- thorough peer review by experienced researchers in your field

- rapid publication on acceptance

- support for research data, including large and complex data types

- gold Open Access which fosters wider collaboration and increased citations

- maximum visibility for your research: over $100 \mathrm{M}$ website views per year

At BMC, research is always in progress.

Learn more biomedcentral.com/submissions 